\title{
Paeoniflorin attenuates the neuroinflammatory response in a rat model of chronic constriction injury
}

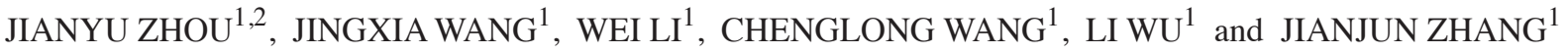 \\ ${ }^{1}$ Department of Traditional Chinese Clinical Pharmacology, \\ School of Preclinical Medicine, Beijing University of Chinese Medicine, Beijing 100029; ${ }^{2}$ Department of \\ Traditional Chinese Medicine, Chengde Medical University, Chengde, Hebei 067000, P.R. China
}

Received January 1, 2016; Accepted December 19, 2016

DOI: $10.3892 / \mathrm{mmr} .2017 .6371$

\begin{abstract}
Neuropathic pain remains the most frequent cause of suffering and disability worldwide. Paeoniflorin (PF), a water-soluble monoterpene glycoside extracted from the roots of Paeonia lactiflora Pall, has a wide range of pharmacological functions. Although the neuroprotective effect of PF has been reported in animal models of neuropathology, no systematic investigation has reported on the analgesic properties of PF in neuropathic pain. The aim of the present study was to investigate whether PF can alleviate neuropathic pain and to examine its possible mechanism. Neuropathic pain was induced by chronic constriction injury (CCI) of the sciatic nerve in rats. Following CCI surgery, the rats were administered with PF for 11 days. Mechanical withdrawal threshold and thermal withdrawal latency were assessed prior to surgery, and on days 3, 7 and 11 post-surgery. The levels of interleukin (IL) $-1 \beta$ and tumor necrosis factor (TNF)- $\alpha$ in the spinal cord were analyzed using enzyme-linked immunosorbent assays. The activation of astrocytes and microglia were observed using immunostaining. In addition, the phosphorylation of p38 mitogen-activated protein kinase (p-p38MAPK) and nuclear factor $-\kappa \mathrm{B}(\mathrm{NF}-\kappa \mathrm{B})$ were examined using western blot analysis. The results indicated that $\mathrm{PF}$ significantly attenuated CCI-induced neuropathic pain and decreased the levels of TNF- $\alpha$ and IL- $1 \beta$ proinflammatory cytokines in the spinal cord. Furthermore, PF inhibited the over-activation of microglia and reduced the elevated expression levels of $\mathrm{p}-\mathrm{p} 38$ MAPK and NF- $\kappa \mathrm{B}$ in the spinal cord. These results indicated that PF offers potential as a therapeutic agent for neuropathic pain, which merits further investigation.
\end{abstract}

Correspondence to: Professor Jianjun Zhang, Department of Traditional Chinese Clinical Pharmacology, School of Preclinical Medicine, Beijing University of Chinese Medicine, 11 Beisanhuadonglu, Chaoyang Qu, Beijing 100029, P.R. China E-mail: 13901024134@163.com

Key words: paeoniflorin, neuropathic pain, microglia, neuroinflammatory, proinflammatory cytokine

\section{Introduction}

Neuropathic pain is a widespread health problem (1). It is a complex disorder, which leads to chronic illness. Although considerable progress has been made, the mechanisms of neuropathic pain remain to be fully elucidated (2). Accumulating evidence indicates that neuroinflammation may be critical in the initiation and maintenance of neuropathic pain, which is now considered to be a neuroimmune disorder (3-6). Studies have shown that the activation of glial cells, including microglia and astrocytes, contributes to central nervous system neuroinflammation and promotes central sensitization, promoting the subsequent development and maintenance of neuropathic pain (7-9). In addition, several studies have shown that inhibiting microglial and astrocytic activation have analgesic effects in neuropathy (10-12). However, no effective drugs have been found to provide efficient treatment.

Chinese herbs are an important resource for potential novel drugs to develop safe and effective agents for the management of neuropathic pain. Several plants have been found to be effective for antagonizing chronic neuropathic pain (13-15). Paeoniae alba Radix, the dried roots of Paeonia lactiflora Pallas or Paeonia veitchii Lynch, is one of the traditional Chinese crude drug herbs. It has been widely used in traditional Chinese prescriptions to alleviate various issues, such as blood extravasation, blood stagnation and female genital diseases (16). Paeoniflorin (Fig. 1), a monoterpene glycoside, is one of the principal active ingredients of Paeoniae alba Radix, and has been reported to exhibit several pharmacological effects, including anti-inflammatory, anti-oxidant and neuroprotective effects (17-20). In nervous disorders in particular, $\mathrm{PF}$ has been reported to exert neuroprotective effects against Alzheimer's disease, cerebral ischemia and Parkinson's disease in experimental models $(18,21,22)$. However, no there have been no reports on the analgesic properties of PF in neuropathic pain.

Previous studies have suggested that PF has potent neuroprotective effects by inhibiting inflammatory responses $(18,19,23,24)$. As neuroinflammation is important in the initiation and maintenance of neuropathic pain, the present study hypothesized that PF can ameliorate neuropathic pain. Therefore, the present study aimed to observed the antagonistic effect of PF on neuropathic pain in a rat model 
of chronic constriction injury (CCI) and to examine its underlying mechanism.

\section{Materials and methods}

Drugs. PF was extracted from Paeoniae alba Radix (Weifang Shengtai Pharmaceutical Industry Co., Ltd., Weifang, China; cat. no. 20150301), and the preparative separation and purification were performed as described previously (25). The purity of PF was determined to be $>98 \%$ using a high-performance liquid chromatographic assay (Fig. 2). PF was dissolved in normal saline solution $(8 \mathrm{mg} / \mathrm{ml})$.

Animals. A total of 30 male Wistar rats (7-week-old; 200-220 g) were obtained from SPF Biotechnology Co., Ltd. (Beijing, China). The rats were housed in a 12-h light/dark schedule at a temperature of $23 \pm 2^{\circ} \mathrm{C}$ and a humidity of $60 \pm 5 \%$ environment with free access to food and water during the 1-week acclimatization period. All animal experiments conformed to the Guide for the Care and Use of Laboratory Animals of the National Institutes of Health (Bethesda, MD, USA). The use of the rats was reviewed and approved by the animal care committee of Beijing University of Chinese Medicine (Beijing, China).

CCI of the sciatic nerve. The animals were subjected to CCI, as previously described by Bennett and Xie (26). In brief, the rats were anesthetized with chloral hydrate $(300 \mathrm{mg} / \mathrm{kg}$ intraperitoneal injection) and the right sciatic nerve was exposed at the mid-thigh level. Proximal to the sciatic trifurcation, adhering tissue was removed from $\sim 7 \mathrm{~mm}$ of the nerve and four ligatures (chromic catgut 4.0) were tied loosely at $1.0 \mathrm{~mm}$ intervals. Sham surgery was performed by exposing the right sciatic nerve without ligation.

Drug treatment. The rats were randomly divided into three groups (10 rats in each group): Sham surgery group (Sham), CCI group (CCI) and CCI+50 mg/kg PF group. The optimal administration dosage of $\mathrm{PF}$ was selected according to the results of preliminary experiments. The PF or vehicle $(10 \mathrm{ml} / \mathrm{kg}$ ) was administered by intraperitoneal injections once a day for 11 days, starting on the first day post-CCI.

Mechanical withdrawal threshold assessment. Mechanical allodynia was examined by assessing the paw withdrawal threshold (PWT) in grams using calibrated Von Frey filaments (North Coast Medical, Inc., Gilroy, CA, USA) as described by Chaplan et al (27). The rats were placed in transparent plexiglass cages on top of an elevated metal mesh floor, and a series of von Frey filaments of logarithmically incremental stiffness were applied using Chaplan's up-down method at the central region of the plantar surface of the right hindpaw, to identify the filament closest to the pain response threshold. Each measurement was repeated three times at intervals of $15 \mathrm{~min}$, and the average force evoking reliable withdrawal was determined as the threshold. This assessment was performed 1 day prior to CCI surgery, and 3, 7 and 11 days post-CCI surgery.

Thermal withdrawal latency assessment. Thermal hyperalgesia was measured using a BME-410C thermal pain stimulator (Institute of Medical Biology, Chinese Academy Of Medical Sciences, Beijing, China) as described previously by Hargreaves et al (28). The rats were placed in transparent plexiglass cages on top of an elevated glass platform, and appropriate intensity radiant heat $\left(55 \pm 0.5^{\circ} \mathrm{C}\right)$ was applied from underneath the platform to the plantar surface of the hindpaw until the rats showed positive signs of pain (licking or withdrawing the paw). The time taken for the rat to lick or withdraw its paw was recorded and defined as the paw withdrawal latency (PWL). A cut-off time of $25 \mathrm{sec}$ was used to prevent tissue damage. Each measurement was repeated three times at intervals of $15 \mathrm{~min}$, and the average force evoking reliable withdrawal was determined as the threshold. This assessment was performed 1 day prior to CCI surgery, and 1, 3, 7 and 11 days post-CCI surgery.

Enzyme-linked immunosorbent assay (ELISA). On the 11th day following CCI surgery, 60 min following the final drug administration (PF or vehicle), the rats were sacrificed by overdose with chloral hydrate $(350 \mathrm{mg} / \mathrm{kg}$ intraperitoneal). The L4-L5 spinal cords ipsilateral to the nerve injury were removed, frozen in liquid nitrogen and then stored at $-80^{\circ} \mathrm{C}$ until further processing. The frozen spinal cords were homogenized in cold phosphate-buffered solution $(10 \mu \mathrm{l} / \mathrm{mg}$ tissue). Following centrifugation at $10,000 \times \mathrm{x}$ for $15 \mathrm{~min}$, the supernatant was used for ELISA. The expression levels of the TNF- $\alpha$ and IL- $1 \beta$ cytokines were measured using ELISA kits (Cusabio Biotech Co., Ltd., Wuhan, China) according to the manufacturer's protocol.

Immunostaining. The L4-L5 spinal cord ipsilateral to the nerve injury was fixed with $4 \%$ paraformaldehyde for $24 \mathrm{~h}$ and paraffin-embedded and cut into $5 \mu \mathrm{m}$ thick sections. The spinal cord sections were deparaffinized following routine methods. Sections were then subjected to antigen retrieval $(\mathrm{pH} 6.0$, citric acid antigen repair buffer) and heated in microwave for $15 \mathrm{~min}$ ( $8 \mathrm{~min}$ at mid-range and $7 \mathrm{~min}$ at low-grade). The sections were blocked using 3\% BSA (cat. no. A8020; Beijing Solarbio Science \& Technology Co., Ltd., Beijing, China) for $30 \mathrm{~min}$ at room temperature. The sections were then incubated with goat polyclonal anti-ionized calcium-binding adapter molecule-1 (Iba-1) antibody (cat. no. ab5076; 1:100; Abcam, Cambridge UK) or rabbit polyclonal anti-glial fibrillary acidic protein (GFAP; cat. no. BA0056; 1:100; Boster Systems, Inc., Pleasanton, CA, USA) at $4^{\circ} \mathrm{C}$ overnight. Following extensive washing in PBS, the sections were incubated with appropriate biotinylated secondary antibodies (cat. nos. GB23204 and GB23303; 1:200 in PBS; Goodbio technology Co., Ltd., Wuhan, China) at room temperature for $1 \mathrm{~h}$, followed by incubation using an SABC immunohistochemistry kit (Goodbio Technology Co., Ltd.) according to the manufacturer's protocol. The microglia and astrocytes were stained and observed using an Image-Pro Plus 6.0 imaging analysis system (Media Cybernetics, Inc., Rockville, MD, USA).

Western blot analysis. The protein was extracted by RIPA lysis buffer (cat. no. P0013C; Beyotime Institute of Biotechnology, Haimen, China). Western blot analysis was used to quantify the protein expression of NF- $\mathrm{NBp} 65$ in the nucleus and the total protein expression of phosphorylated (p-) p38 


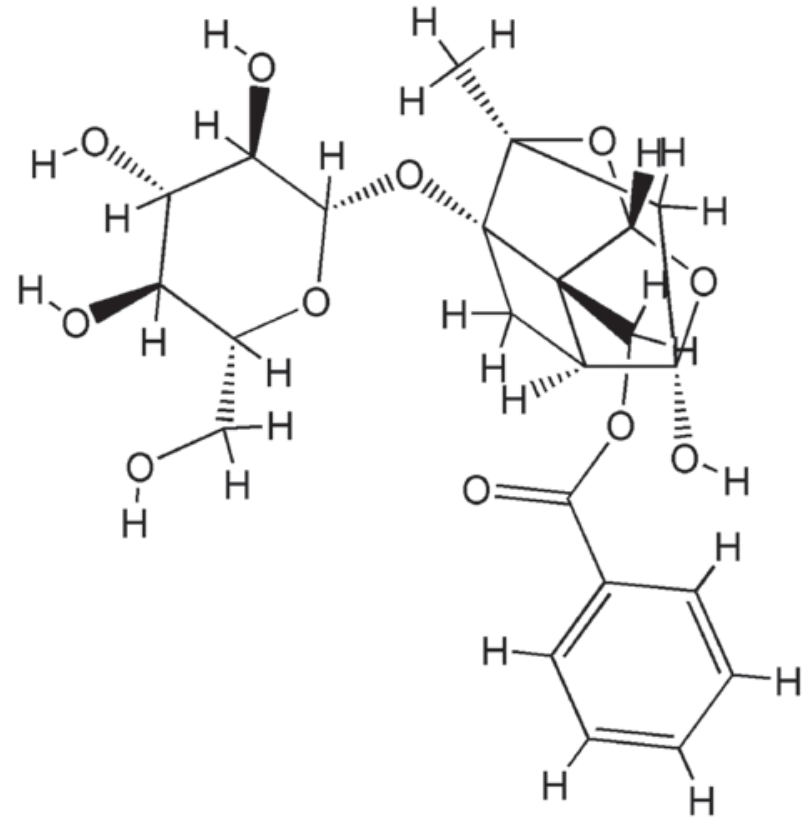

Figure 1. Chemical structure of peaoniflorin.

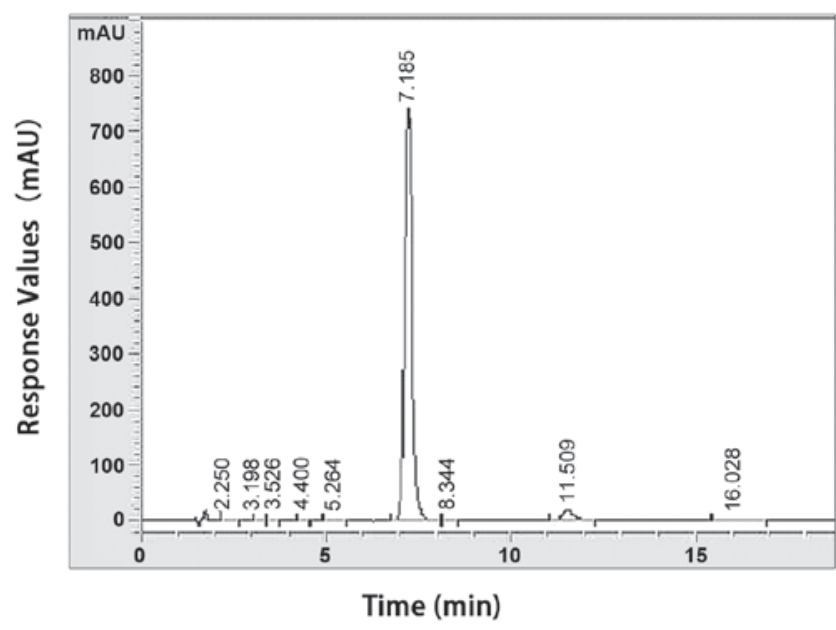

Figure 2. High-performance liquid chromatography chromatogram of paeoniflorin.

mitogen-activated protein kinase (MAPK)/p38MAPK in the total protein extracted from the spinal cord. The concentration of the protein was examined by BCA method using protein quantitative kit (cat. no. P0012; Beyotime Institute of Biotechnology) An equal quantity of protein $(50 \mu \mathrm{g})$ was loaded and separated by $12 \%$ SDS-PAGE. The resolved proteins were transferred onto nitrocellulose membranes (EMD Millipore, Billerica, MA, USA). The membranes were then blocked in 5\% nonfat milk for $2 \mathrm{~h}$ at room temperature and incubated overnight at $4^{\circ} \mathrm{C}$ with mouse anti-NF-kBp65 (cat. no. CST6956; 1:1,000; Cell Signaling Technology, Inc., Danvers, MA, USA), rabbit anti-p-p38MAPK (cat. no. CST4631; 1:1,000; Cell Signaling Technology, Inc.) and rabbit anti-p38MAPK (cat. no. CST8690; 1:1,000; Cell Signaling Technology, Inc.) respectively. The blots were then incubated with goat anti-mouse and goat anti-rabbit secondary antibodies (horseradish peroxidase; Goodbio Technology Co., Ltd.) conjugated with horseradish peroxidase (1:500, ZSGB-BIO, Beijing, China) for $1 \mathrm{~h}$ at room temperature. The protein bands were visualized with chemiluminescence reagent (Engreen Biosystem, Ltd., Beijing, China). Western blot densitometry analysis of signal intensity was performed using Image-Pro Plus imaging analysis system (version, 6.0; Media Cybernetics, Inc., Rockville, MD, USA).

Statistical analysis. The effect of each treatment was analyzed using SPSS 17.0 software (SPSS, Inc., Chicago, IL, USA). All data are expressed as the mean \pm standard error of the mean. Statistical analysis was performed using one-way analysis of variance, followed by the least-significant difference post hoc test or Dunnett's T3 test for comparison of multiple groups. $\mathrm{P}<0.05$ was considered to indicate a statistically significant difference.

\section{Results}

$P F$ attenuates increased $P W T$ and $P W L$ in CCI rats. The effects of PF on PWT and PWL in CCI rats are shown in Fig. 3A and B. Prior to CCI surgery, no significant differences were found in the baseline PWL and PWT among all groups. PWT and PWL in the CCI groups decreased markedly 3,7 and 11 days post-CCI $(\mathrm{P}<0.001)$, compared with those in the sham groups, indicating that CCI induced long-lasting thermal hyperalgesia and mechanical allodynia. Following PF administration, the PWL and PWT were significantly increased in the rats, compared with those in the CCI group, on day 11 (PWT; P $<0.001$; PWL, $\mathrm{P}<0.05$ ). These results demonstrated that $\mathrm{PF}$ produced an antinociceptive effect on CCI-induced pain, including mechanical and thermal hyperalgesia, 11 days following CCI.

Anti-inflammatory effects of $P F$ in CCI rats. To investigate the effects of PF on CCI-induced neuroinflammation, the levels of proinflammatory cytokines, IL- $1 \beta$ and TNF- $\alpha$, in spinal cord samples were determined. As shown in Fig. 4A and B, compared with the sham group, the levels of IL- $1 \beta$, and TNF- $\alpha$ were markedly increased in the spinal cord of the CCI rats $(\mathrm{P}<0.001)$. $\mathrm{PF}$ treatment significantly decreased these elevated proinflammatory cytokine levels, compared with the levels in the CCI groups (TNF- $\alpha, \mathrm{P}<0.01$; IL-1 $\beta, \mathrm{P}<0.05$ ).

$P F$ inhibits the activation of microglia in CCI rats. To examine the possible mechanisms underlying the protective effects of $\mathrm{PF}$ on neuropathic pain in rats, the activation of astrocytes and microglia were monitored. As shown in Fig. 5A-D, compared with the sham group, the expression levels of the astrocyte marker (GFAP) and microglial marker (Iba-1) were increased significantly in the CCI rats $(\mathrm{P}<0.05)$. However, compared with the CCI group, the expression of Iba- $1(\mathrm{P}<0.05)$, but not GFAP $(\mathrm{P}>0.05)$ decreased significantly in the $\mathrm{CCI}+\mathrm{PF}$ group, suggesting that $\mathrm{PF}$ inhibited the activation of microglia but not astrocytes.

Effects of PF on the activation of $p 38 M A P K$ in the spinal cord of CCI rats. To further examine the mechanisms underlying the effect of $\mathrm{PF}$, the present study investigated the expression of p-p38 in the spinal cord. As shown in Fig. 6A and B, the expression level of p-p38 was significantly increased in the CCI group, compared with that in the sham group $(\mathrm{P}<0.05)$. 

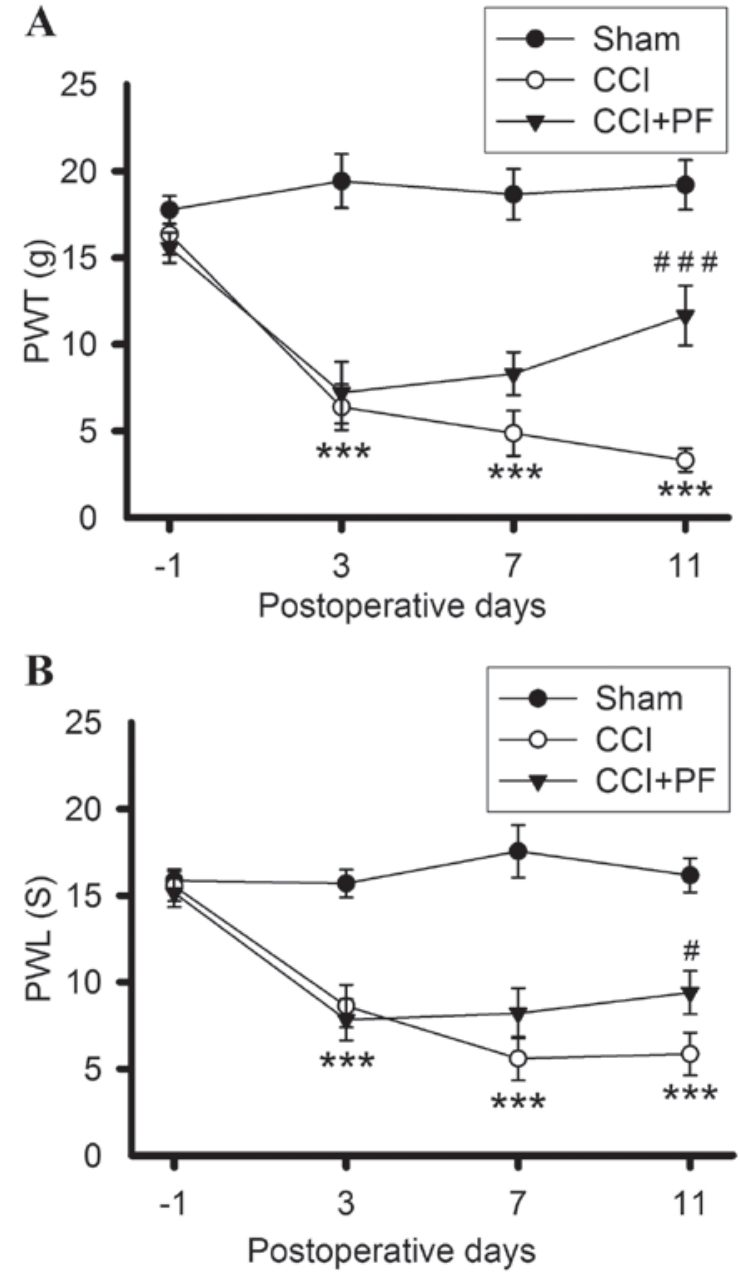

Figure 3. Effects of PF on PWT and PWL in CCI rats. (A) PWT was determined using a von Frey filament test and (B) PWL was measured using a thermal pain stimulator. Data are expressed as the mean \pm standard error of the mean $(\mathrm{n}=10) .{ }^{* * *} \mathrm{P}<0.001$, compared with the Sham group; ${ }^{*} \mathrm{P}<0.05$ and ${ }^{\# \# \#} \mathrm{P}<0.001$, compared with the CCI group. PWT, paw withdrawal threshold; PWL, paw withdrawal latency; CCI, chronic constriction injury; $\mathrm{PF}$, peaoniflorin.

PF treatment markedly decreased the elevated level of p-p38 observed in the CCI group $(\mathrm{P}<0.05)$. These results demonstrated that PF inhibited the p38MAPK pathway.

Effects of PF on the activation of $N F-\kappa B$ in the spinal cord of CCI rats. NF- $\kappa \mathrm{B}$ is a crucial transcription factor complex controlling the expression of proinflammatory and pain mediators. To investigate the mechanism underlying the analgesic effect of $\mathrm{PF}$, the present study monitored the expression of NF- $\mathrm{\kappa Bp} 65$, a nuclear protein associated with the NF- $\mathrm{\kappa B}$ signaling pathway, in the spinal cord of the CCI rats. As shown in Fig. 6C, compared with the sham group, the expression level of nuclear NF-kBp65 was significantly increased by $\mathrm{CCI}(\mathrm{P}<0.01)$. PF significantly decreased the protein expression of NF- $\mathrm{\kappa Bp} 65$ in the spinal cord, compared with that in the CCI group $(\mathrm{P}<0.05)$. These results indicated that PF inhibited the NF-kB pathway.

\section{Discussion}

The present study demonstrated for the first time, to the best of our knowledge, that PF alleviated the neuropathic pain induced
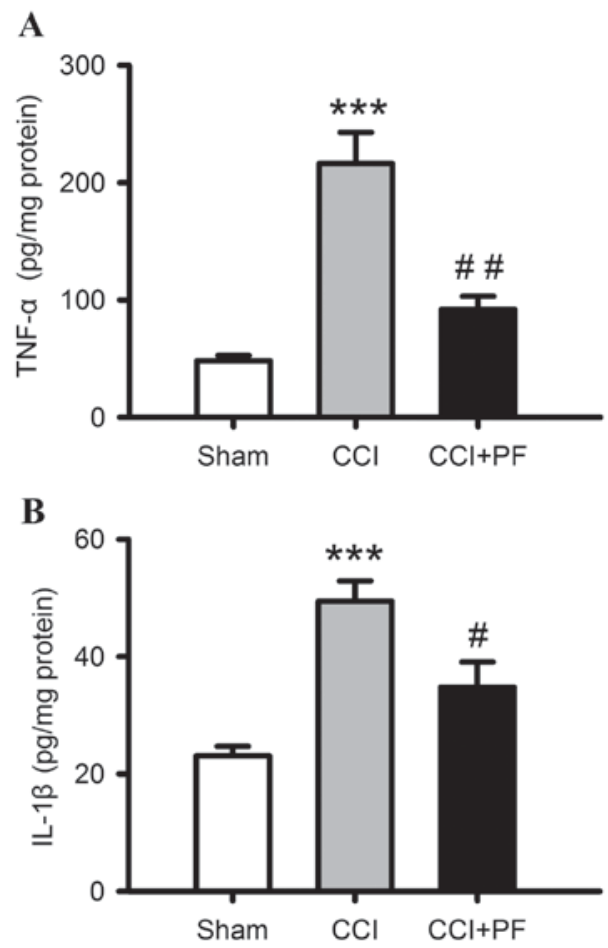

Figure 4. Effects of $\mathrm{PF}$ on elevated proinflammation in the spinal card of $\mathrm{CCI}$ rats. Following treatment with PF for 11 days post-CCI, the levels of TNF- $\alpha$ and IL-1 $\beta$ were measured using an enzyme-linked immunosorbent assay. (A) Expression of TNF- $\alpha$ in the spinal cord. (B) Expression of IL- $1 \beta$ in the spinal card. Data assessment was performed in triplicate, $(\mathrm{n}=4) .{ }^{* * * *} \mathrm{P}<0.001$, compared with the Sham group; ${ }^{\#} \mathrm{P}<0.05$ and ${ }^{\# \#} \mathrm{P}<0.01$, compared with the CCI group. $\mathrm{CCI}$, chronic constriction injury; PF, peaoniflorin; TNF- $\alpha$, tumor necrosis factor- $\alpha$; IL-1 $\beta$, interleukin- $1 \beta$.

by $\mathrm{CCI}$ in rats. It was found that PF attenuated CCI-induced neuropathic pain, including mechanical and thermal hyperalgesia, and decreased expression levels of the TNF- $\alpha$ and IL-1 $\beta$ proinflammatory cytokines in the spinal cord. In addition, PF inhibited the activation of microglia and reduced the elevated expression of p-p38 MAPK/NF- $\mathrm{kB}$ in the spinal cord induced by $\mathrm{CCI}$. These results suggested that $\mathrm{PF}$ offers potential for use as a therapeutic agent for neuropathic pain.

The CCI model is a widely used rodent model to investigate neuropathic pain mechanisms (29-31). This model successfully produces long-lasting thermal hyperalgesia and mechanical allodynia, similar to human behavioral responses $(32,33)$. In the present study, it was found that CCI produced marked mechanical allodynia and thermal hyperalgesia in rats. However, administration of PF for 11 days attenuated mechanical allodynia and thermal hyperalgesia, suggesting the possible therapeutic efficacy of PF. In addition, PF was observed to have a more marked analgesic effect in thermal hyperalgesia, compared with mechanical allodynia. Further investigations are required to examine the underlying mechanism.

Increasing evidence has indicated that proinflammatory cytokines are a critical factor in the initiation and maintenance of hyperalgesia in animal models of neuropathic pain (4). The proinflammatory cytokine-mediated process during neuroinflammation can be induced by nerve injury (5). The CCI model induces the upregulation of proinflammatory cytokines, including IL-1 $\beta$, IL-6 and TNF- $\alpha$, in the spinal cord $(34,35)$. The increase of proinflammatory cytokines in the spinal 
A
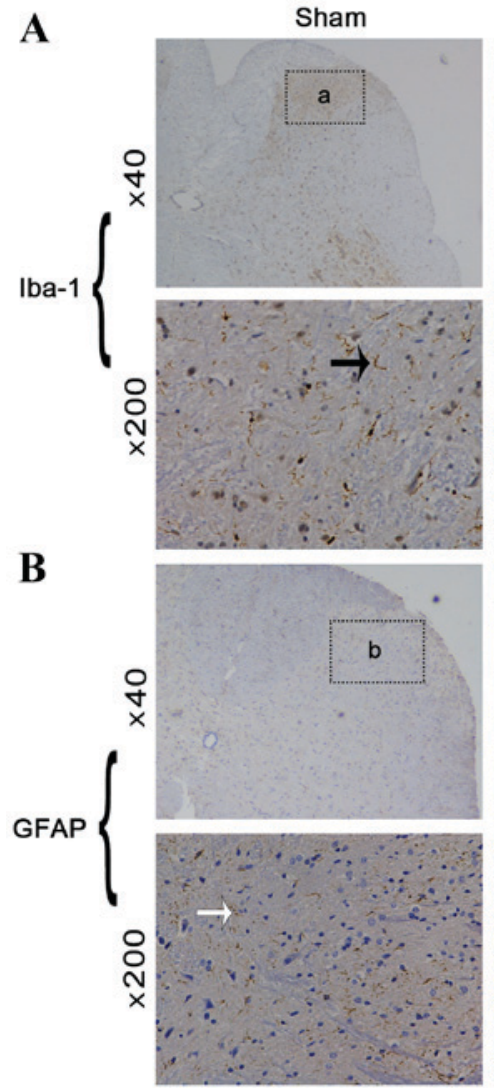

C

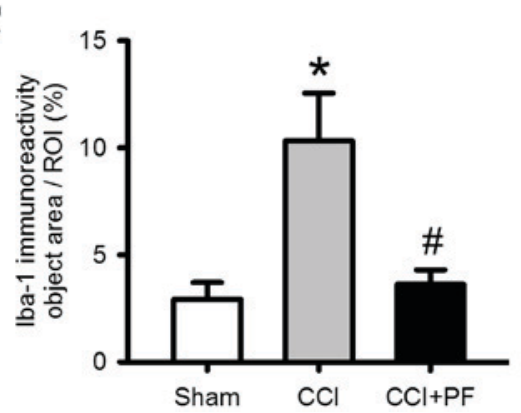

$\mathrm{CCl}$
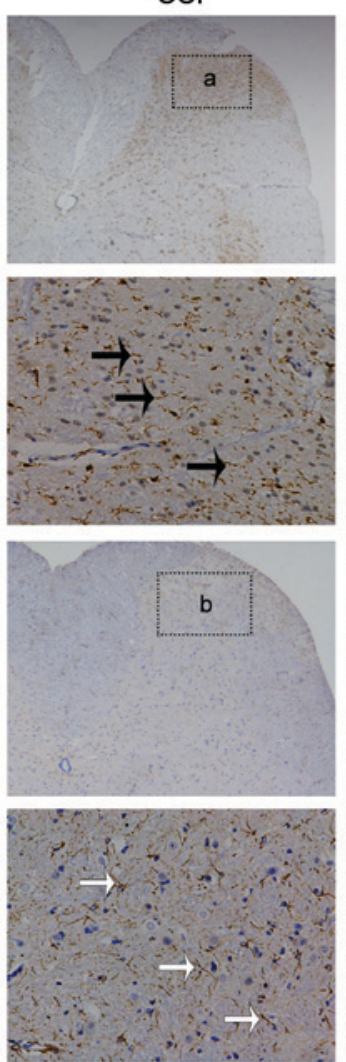

D
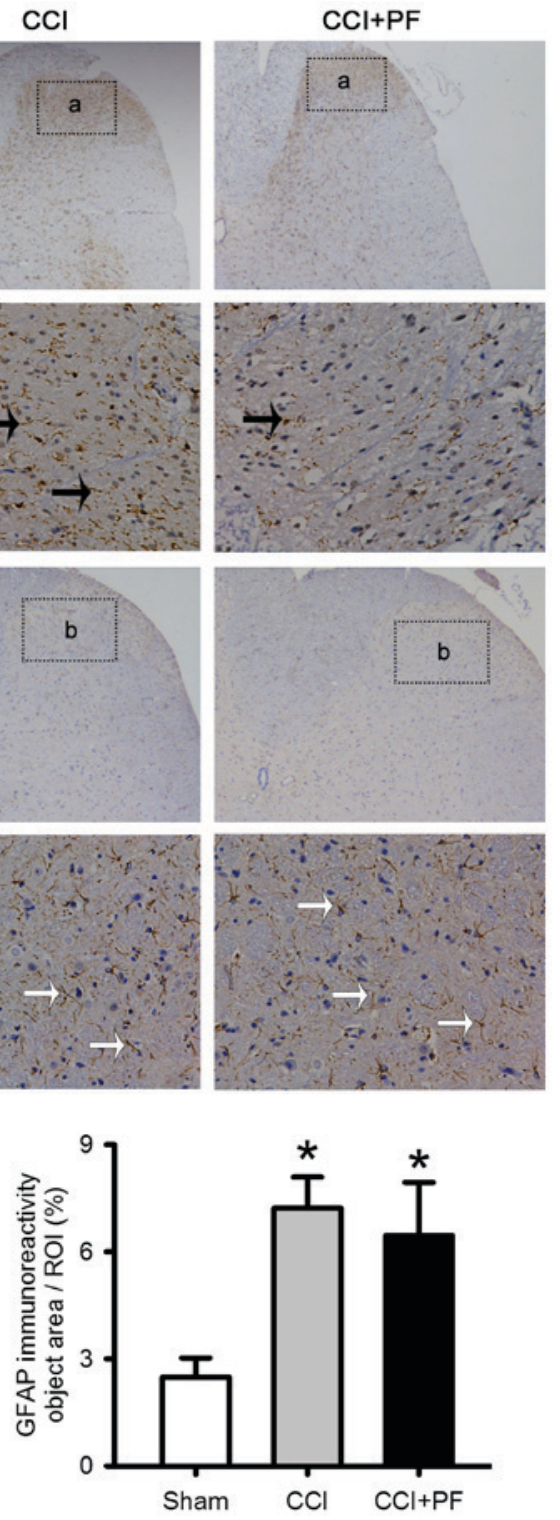

Figure 5. Effects of PF on the activation of microglia and astrocytes in the L4-L5 lumbar segment of CCI rats. Representative images of spinal cord sections stained with astrocyte marker, GFAP, and microglial marker, Iba-1, antibodies. (A) Iba-1 staining in the spinal card; black arrows indicate Iba-1-positive microglia; (a) regions selected for quantitative analysis of microglial marker activation. (B) GFAP staining in the spinal cord; white arrows indicate GFAP-positive astrocytes; (b) regions selected for quantitative analysis of astrocyte marker activation. (C) Quantification of the effect of PF on the microglial marker Iba-1. (D) Quantification of the effect of PF on the astrocyte marker GFAP. Data are expressed as the mean \pm standard error of the mean ( $\mathrm{n}=3$ ). ${ }^{*} \mathrm{P}<0.05$, compared with the Sham group; ${ }^{*} \mathrm{P}<0.05$, compared with the CCI group. CCI, chronic constriction injury; $\mathrm{PF}$, peaoniflorin; GFAP, glial fibrillary acidic protein; Iba-1, ionized calcium-binding adapter molecule-1.

A

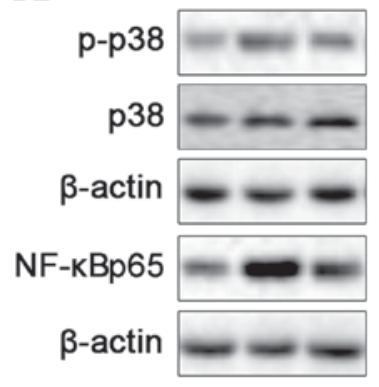

B

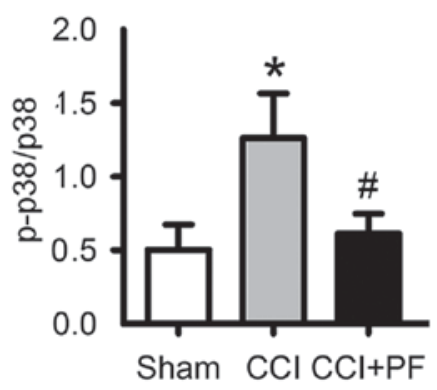

C

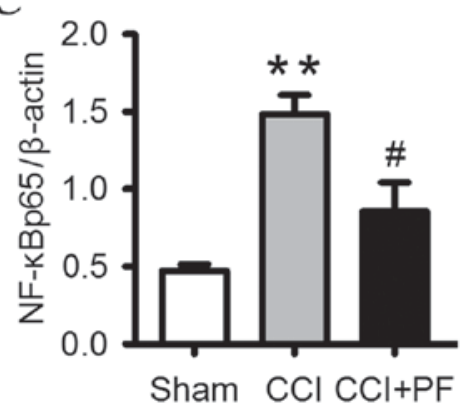

Figure 6. Effects of PF on the expression levels of p-p38 and NF-kBp65 in the spinal cord of CCI rats. Western blot analysis of protein expression levels of

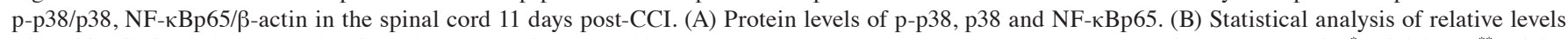
of p-p38. (C) Statistical analysis of relative levels of NF-kBp65. Data are expressed as the mean \pm standard error of the mean $(n=3)$. "P<0.05 and ${ }^{* *} \mathrm{P}<0.01$, compared with the Sham group; ${ }^{*} \mathrm{P}<0.05$, compared with the CCI group. $\mathrm{CCI}$, chronic constriction injury; $\mathrm{PF}$, peaoniflorin; $\mathrm{p}-$, phosphorylated; NF- $\mathrm{kB}$, nuclear factor- $\mathrm{kB}$. 
cord promotes the transduction of detrimental signals by increasing excitatory synaptic transmission and decreasing inhibitory synaptic transmission (36). In the present study, it was found that PF significantly inhibited the overexpression of spinal IL- $1 \beta$ and TNF- $\alpha$ in the CCI rat model. These results indicated $\mathrm{PF}$ as a potential candidate to control neuroinflammation-induced pain.

Following peripheral nerve injury, sensitized primary afferent terminals release nociceptive neurotransmitters and mediators, including glutamate, substance $\mathrm{P}$ and fractalkine, which activate spinal microglia and astrocytes (37). Activated microglia and astrocytes contribute to neuroinflammation, accelerate facilitatory pain transmission, and contribute to the subsequent development and maintenance of neuropathic pain (7-9). The results of the present study showed that spinal astroglia and microglia were markedly activated in the CCI injury model, whereas administration of PF for 11 days inhibited the activation of microglia, suggesting that the activities of PF involved regulation of the spinal glial-neuroimmune system.

MAPKs are a crucial molecules in cell signaling, which consist of p38MAPK, extracellular signal-related kinase 1/2 and c-Jun amino terminal kinase 1/2 (38). Emerging evidence has indicates that nerve injury results in the activation of p38MAPK in the spinal cord, and p38MAPK regulates the production of proinflammatory cytokines to promote the development of neuropathic pain (39-41). In addition, several studies have suggested that p38MAPK is critical in microglial signaling under neuropathic pain conditions and represents a valuable therapeutic target for neuropathic pain $(42,43)$. The results of the present study indicated that PF treatment prevented the CCI-induced upregulation in the protein level of p-p38 when measured 11 days post-nerve injury. The same trend was observed in the effect of PF on the inhibition of CCI-induced activation and release of proinflammatory cytokines. These results suggested that PF-mediated inhibition of CCI-induced p38MAPK activation is a possible mechanism underlying its inhibitory action on neuropathic pain.

$\mathrm{NF}-\kappa \mathrm{B}$, a pleiotropic factor, which regulates several physiological processes and is important in regulating the immune response (44). Previous studies have demonstrated that the activation of $\mathrm{NF}-\kappa \mathrm{B}$ occurs in the spinal cord, which is involved in the transmission and processing of nociceptive information. Following activation, NF- $\mathrm{B}$ transfers into the nucleus and regulates the synthesis and release of proinflammatory cytokines, including IL-1 $\beta$, IL- 6 and TNF- $\alpha$, which may be crucial in neuroinflammation $(45,46)$. It has been reported that the administration of $\mathrm{NF}-\kappa \mathrm{B}$ inhibitors exerts an analgesic effect in various animal pain models $(47,48)$. In the present study, it was found that PF reduced the CCI-elevated expression of $\mathrm{NF}-\kappa \mathrm{B}$ in the spinal cord. In addition, $\mathrm{PF}$ inhibited the activation of $\mathrm{NF}-\kappa \mathrm{B}$ and the subsequent expression of proinflammatory cytokines, which may be beneficial for reducing neuropathic pain.

In conclusion, the results of the present study demonstrated that PF produced a significant analgesic action in CCI-injury rats and that this activity was associated with the modulation of neuroinflammation in the spinal cord. These results suggested that $\mathrm{PF}$ is a potential therapeutic agent for neuropathic pain, which merits further investigation.

\section{Acknowledgements}

This study was supported by the National Natural Science Foundation of China (grant nos. 81473370 and 81173569).

\section{References}

1. Gilron I, Watson CP, Cahill CM and Moulin DE: Neuropathic pain: A practical guide for the clinician. CMAJ 175: 265-275, 2006.

2. Cornelius VR, Sauzet O, Williams JE, Ayis S, Farquhar-Smith P, Ross JR, Branford RA and Peacock JL: Adverse event reporting in randomised controlled trials of neuropathic pain: Considerations for future practice. Pain 154: 213-220, 2013.

3. Streit WJ, Mrak RE and Griffin WS: Microglia and neuroinflammation: A pathological perspective. J Neuroinflammation 1: 14, 2004.

4. Moalem G and Tracey DJ: Immune and inflammatory mechanisms in neuropathic pain. Brain Res Rev 51: 240-264, 2006.

5. Myers RR, Campana WM and Shubayev VI: The role of neuroinflammation in neuropathic pain: Mechanisms and therapeutic targets. Drug Discov Today 11: 8-20, 2006.

6. Wahba $\mathrm{M}$ and Waln O: Asterixis related to gabapentin intake: A case report and review. Postgrad Med 125: 139-141, 2013.

7. Marchand F, Perretti M and McMahon SB: Role of the immune system in chronic pain. Nat Rev Neurosci 6: 521-532, 2005.

8. Milligan ED and Watkins LR: Pathological and protective roles of glia in chronic pain. Nat Rev Neurosci 10: 23-36, 2009.

9. Bradesi S: Role of spinal cord glia in the central processing of peripheral pain perception. Neurogastroenterol Motil 22: 499-511, 2010.

10. Sweitzer SM, Schubert P and DeLeo JA: Propentofylline, a glial modulating agent, exhibits antiallodynic properties in a rat model of neuropathic pain. J Pharmacol Exp Ther 297: 1210-1217, 2001.

11. Jean YH, Chen WF, Sung CS, Duh CY, Huang SY, Lin CS, Tai MH, Tzeng SF and Wen ZH: Capnellene, a natural marine compound derived from soft coral, attenuates chronic constriction injury-induced neuropathic pain in rats. Br J Pharmacol 158: 713-725, 2009.

12. Lin YC, Huang SY, Jean YH, Chen WF, Sung CS, Kao ES, Wang HM, Chakraborty C, Duh CY and Wen ZH: Intrathecal lemnalol, a natural marine compound obtained from Formosan soft coral, attenuates nociceptive responses and the activity of spinal glial cells in neuropathic rats. Behav Pharmacol 22: 739-750, 2011.

13. Kim YS, Park HJ, Kim TK, Moon DE and Lee HJ: The effects of Ginkgo biloba extract EGb 761 on mechanical and cold allodynia in a rat model of neuropathic pain. Anesth Analg 108: 1958-1963, 2009.

14. Gao T, Hao J, Wiesenfeld-Hallin Z, Wang DQ and Xu XJ: Analgesic effect of sinomenine in rodents after inflammation and nerve injury. Eur J Pharmacol 721: 5-11, 2013.

15. Zhou X, Cheng H, Xu D, Yin Q, Cheng L, Wang L, Song S and Zhang M: Attenuation of neuropathic pain by saikosaponin a in a rat model of chronic constriction injury. Neurochem Res 39: 2136-2142, 2014

16. Wu SH, Wu DG and Chen YW: Chemical constituents and bioactivities of plants from the genus Paeonia. Chem Biodivers 7: 90-104, 2010.

17. Zhong SZ, Ge QH, Li Q, Qu R and Ma SP: Peoniflorin attentuates Abeta(1-42)-mediated neurotoxicity by regulating calcium homeostasis and ameliorating oxidative stress in hippocampus of rats. J Neurol Sci 280: 71-78, 2009.

18. Guo RB, Wang GF, Zhao AP, Gu J, Sun XL and $\mathrm{Hu}$ G: Paeoniflorin protects against ischemia-induced brain damages in rats via inhibiting MAPKs/NF- $\kappa \mathrm{B}$-mediated inflammatory responses. PLoS One 7: e49701, 2012.

19. Nam KN, Yae CG, Hong JW, Cho DH, Lee JH and Lee EH: Paeoniflorin, a monoterpene glycoside, attenuates lipopolysaccharide-induced neuronal injury and brain microglial inflammatory response. Biotechnol Lett 35: 1183-1189, 2013.

20. Wu YM, Jin R, Yang L, Zhang J, Yang Q, Guo YY, Li XB, Liu SB, Luo XX and Zhao MG: Phosphatidylinositol 3 kinase/protein kinase $\mathrm{B}$ is responsible for the protection of paeoniflorin upon $\mathrm{H}_{2} \mathrm{O}_{2}$-induced neural progenitor cell injury. Neuroscience 240: 54-62, 2013. 
21. Liu HQ, Zhang WY, Luo XT, Ye Y and Zhu XZ: Paeoniflorin attenuates neuroinflammation and dopaminergic neurodegeneration in the MPTP model of Parkinson's disease by activation of adenosine A1 receptor. Br J Pharmacol 148: 314-325, 2006.

22. Zhang HR, Peng JH, Cheng XB, Shi BZ, Zhang MY and Xu RX: Paeoniflorin atttenuates amyloidogenesis and the inflammatory responses in a transgenic mouse model of Alzheimer's disease. Neurochem Res 40: 1583-1592, 2015.

23. Dong H, Li R, Yu C, Xu T, Zhang X and Dong M: Paeoniflorin inhibition of 6-hydroxydopamine-induced apoptosis in PC12 cells via suppressing reactive oxygen species-mediated $\mathrm{PKC} / \mathrm{NF}-\kappa \mathrm{B}$ pathway. Neuroscience 285: 70-80, 2015.

24. Liu H, Wang J, Wang J, Wang P and Xue Y: Paeoniflorin attenuates $A \beta 1-42$-induced inflammation and chemotaxis of microglia in vitro and inhibits NF- $\mathrm{KB}$ - and VEGF/Flt-1 signaling pathways. Brain Res 1618: 149-158, 2015.

25. Chen F, Lu HT and Jiang Y: Purification of paeoniflorin from Paeonia lactiflora Pall. By high-speed counter-current chromatography. J Chromatogr A 1040: 205-208, 2004.

26. Bennett GJ and Xie YK: A peripheral mononeuropathy in rat that produces disorders of pain sensation like those seen in man. Pain 33: 87-107, 1988.

27. Chaplan SR, Bach FW, Pogrel JW, Chung JM and Yaksh TL: Quantitative assessment of tactile allodynia in the rat paw. J Neurosci Methods 53: 55-63, 1994.

28. Hargreaves K, Dubner R, Brown F, Flores C and Joris J: A new and sensitive method for measuring thermal nociception in cutaneous hyperalgesia. Pain 32: 77-88, 1988

29. Vierck CJ, Acosta-Rua AJ and Johnson RD: Bilateral chronic constriction of the sciatic nerve: A model of long-term cold hyperalgesia. J Pain 6: 507-517, 2005.

30. Jaggi AS, Jain V and Singh N: Animal models of neuropathic pain. Fundam Clin Pharmacol 25: 1-28, 2011

31. Chu LW, Chen JY, Yu KL, Cheng KI, Wu PC and Wu BN Neuroprotective and anti-inflammatory activities of atorvastatin in a rat chronic constriction injury model. Int J Immunopathol Pharmacol 25: 219-230, 2012.

32. Wang LX and Wang ZJ: Animal and cellular models of chronic pain. Adv Drug Deliv Rev 55: 949-965, 2003.

33. Xu Y, Qiu HQ, Liu H, Liu M, Huang ZY, Yang J, Su YP and Yu CX: Effects of koumine, an alkaloid of Gelsemium elegans Benth., on inflammatory and neuropathic pain models and possible mechanism with allopregnanolone. Pharmacol Biochem Behav 101: 504-514, 2012.

34. Costa B, Trovato AE, Colleoni M, Giagnoni G, Zarini E and Croci T: Effect of the cannabinoid CB1 receptor antagonist, SR141716, on nociceptive response and nerve demyelination in rodents with chronic constriction injury of the sciatic nerve. Pain 116: 52-61, 2005

35. Kiguchi N, Kobayashi Y and Kishioka S: Chemokines and cytokines in neuroinflammation leading to neuropathic pain. Curr Opin Pharmacol 12: 55-61, 2012.
36. Kawasaki Y, Zhang L, Cheng JK and Ji RR: Cytokine mechanisms of central sensitization: Distinct and overlapping role of interleukin-1beta, interleukin-6, and tumor necrosis factor-alpha in regulating synaptic and neuronal activity in the superficial spinal cord. J Neurosci 28: 5189-5194, 2008.

37. Cao $\mathrm{H}$ and Zhang YQ: Spinal glial activation contributes to pathological pain states. Neurosci Biobehav Rev 32: 972-983, 2008.

38. Chang L and Karin M: Mammalian MAP kinase signalling cascades. Nature 410: 37-40, 2001

39. Obata K, Yamanaka H, Kobayashi K, Dai Y, Mizushima T, Katsura H, Fukuoka T, Tokunaga A and Noguchi K: Role of mitogen-activated protein kinase activation in injured and intact primary afferent neurons for mechanical and heat hypersensitivity after spinal nerve ligation. J Neurosci 24: 10211-10222, 2004.

40. Xu JT, Xin WJ, Wei XH, Wu CY, Ge YX, Liu YL, Zang Y, Zhang T, Li YY and Liu XG: p38 activation in uninjured primary afferent neurons and in spinal microglia contributes to the development of neuropathic pain induced by selective motor fiber injury. Exp Neurol 204: 355-365, 2007.

41. Xu L, Huang Y, Yu X, Yue J, Yang N and Zuo P: The influence of p38 mitogen-activated protein kinase inhibitor on synthesis of inflammatory cytokine tumor necrosis factor alpha in spinal cord of rats with chronic constriction injury. Anesth Analg 105: 1838-1844, table of contents, 2007

42. Ji RR and Suter MR: p38 MAPK, microglial signaling, and neuropathic pain. Mol Pain 3: 33, 2007.

43. Rojewska E, Popiolek-Barczyk K, Jurga AM, Makuch W, Przewlocka B and Mika J: Involvement of pro- and antinociceptive factors in minocycline analgesia in rat neuropathic pain model. J Neuroimmunol 277: 57-66, 2014.

44. Sun T, Song WG, Fu ZJ, Liu ZH, Liu YM and Yao SL: Alleviation of neuropathic pain by intrathecal injection of antisense oligonucleotides to p65 subunit of NF-kappaB. Br J Anaesth 97: 553-558, 2006

45. Makarov SS: NF-kappaB as a therapeutic target in chronic inflammation: Recent advances. Mol Med Today 6: 441-448, 2000.

46. Niederberger E and Geisslinger G: The IKK-NF-kappaB pathway: A source for novel molecular drug targets in pain therapy. FASEB J 22: 3432-3442, 2008.

47. Laughlin TM, Bethea JR, Yezierski RP and Wilcox GL: Cytokine involvement in dynorphin-induced allodynia. Pain 84: 159-167, 2000.

48. Wei XH, Yang T, Wu Q, Xin WJ, Wu JL, Wang YQ, Zang Y, Wang J, Li YY and Liu XG: Peri-sciatic administration of recombinant rat IL-1 $\beta$ induces mechanical allodynia by activation of src-family kinases in spinal microglia in rats. Exp Neurol 234: 389-397, 2012. 